\title{
Application of mathematical approach to the psychological aspects of human
}

\author{
2021/06/13 Second Edition
}

Takuya Yabu(takuya.yabu@live.jp)

\begin{abstract}
I took a mathematical approach to the psychological aspects of humans. Apply the result to another psychological phenomenon. First, I examined cognition before humans feel emotions, and found the probability density function for cognition. I showed that humans can be classified into four patterns by dividing cognition and emotion. Also, in order to live normally in a group, I found a differential equation satisfied by a probability distribution function that expresses how individual's emotional distribution looks to the group, and found a solution. As a result, I found the initial conditions that give the standard normal distribution, which is the probability density function of the emotions of the group, and showed how the probability distribution function that looks like a group becomes the same standard normal distribution as the group. Using this result, I showed how to live normally without being excluded in the group. The method shown was also pointed out in cognitive-behavioral therapy, and the same results as those obtained from many cases were obtained. Also, even if you have difficulty living, think mathematically in the same way as how to prevent being excluded from the group, and show how individual behave toward oneself. The method shown was also pointed out in cognitive-behavioral therapy, and the same results as those obtained from many cases were obtained
\end{abstract}

\section{Introduction}

I took a mathematical approach to the psychological aspects of humans (Yabu, 2020). The results obtained there are applied to other psychological phenomena. First, I consider cognition before humans feel emotions. Various studies have been conducted on cognition in the past. The decision field theory mathematically explained how human decide things (Busemeyer \& Townsend, Decision Field Theory: A dynamic cognition approach to decision making, 1993) (Busemeyer \& Diederich, Survey of decision field theory, 2002). That is the part of cognition. Recently, human cognitive process is applied many fields. The Cognitive Affective Model of Immersive Learning (CAMIL), which synthesized existing immersive educational research to describe the process of learning in immersive virtual reality (IVR) was explained (Makransky \& Petersen, 2021). Wu et al. explained a better understanding of the mastery of students of fine-grained knowledge points through the Program for International Student Assessment (PISA) 
(Xiaopeng, Rongxiu, Hua-Hua, Qiping, \& Yi, 2020). Lena and Adele reviewed the field of multi-alternative multi-attribute decision-making research including cognitive process models (Wollschlaeger \& Diederich, 2020). Zandor et al. proposed a model, and implementation of the cognitive function of imitation motor learning (IML), based on psychological and neuroscientific evidence and they applied to motor learning (Machaena, Martinb, \& Rosalesa, 2021). Andrei et al. proposed a brain-inspired cognitive support model for stress reduction based on an adaptive network model and implemented and tested (Andrianov, Ziabari, \& Gerritsen, 2021). Nada et al. disclosed how cognitive processes influence script concordance test responses (Gawad, Wood, Cowley, \& Raiche, 2021). Yunfei and Carol developed a cognitive modeling and simulation environment, CMS-BN, for human operator performance assessment (Yunfei \& Smidts, 2021). However, as far as the author knows, there is no example of a mathematical approach. As with the reference (Yabu, 2020), I consider cognition using a one-dimensional random walk or Wiener process, and obtain a probability density function for cognition. As a result, it is shown that human beings can be classified into 4 types because the probability of being in one of the two types is very high for each of cognition and emotion. Next, I show how to behave in order to live normally in a group. Various studies have also been conducted on psychology within the group. Carin and Joris explained how the social environment influences payment behavior using individual's payment diary data (van der Cruijsen \& Knoben , 2021). Shilpi and Jayashree presented a step-by-step guide to facilitate understanding of emerging trends in the discipline of Organizational Behavior using the technique of Systematic Literature Review (Kalwani \& Mahesh, 2020). Keith et al. showed effective classroom behavior management which approach individual's action increase student achievement in middle school (Herman, Reinke, Dong, \& Bradshaw, 2020). Monge and O'Brien researched individual toxic behavior in a online video game and disclosed toxic behavior significantly worsened the team and individual performance (Monge \& O’Brien, 2021). Antonia and Yarrow researched group influence on children's prosocial and antisocial behavior and indicated children were not blindly conformist and imitate more prosocial than antisocial behavior (Misch \& Dunham, 2021). However, as far as the author knows, there is no example of a mathematical approach. To that end, consider a probability distribution function that expresses what the probability distribution of one's emotions looks like to a group. Find the differential equation that the probability distribution function satisfies and find the solution. As a result, I find the initial conditions that give the standard normal distribution, which is the probability density function of the emotions of the group, and show how the probability 
distribution function that looks like a group becomes the same standard normal distribution as the group. Using this result, I show how to live normally without being excluded in the group. The method shown was also pointed out in cognitive-behavioral therapy, and the same results as those obtained from many cases were obtained. Also, even if individual have difficulty living, think mathematically in the same way as how to prevent being excluded from the group, and show how individual behave toward oneself. Various studies have also been conducted on difficulty living. Gong and Chao researched difficulty in activities of daily living among postmenopausal women (Gong \& $\mathrm{Wu}, 2021$ ). Kaitlyn et al. disclosed difficulty living of adults with fetal alcohol spectrum disorder (McLachlan, Flannigan, Temple, Unsworth, \& Cook, 2020). Lisa et al. revealed relationship between depression and suicidal ideation in older prisoners (Barrya, et al., 2020). However, as far as the author knows, there is no example of a mathematical approach. The method shown was also pointed out in cognitive-behavioral therapy, and the same results as those obtained from many cases were obtained.

\section{Mathematical approach to cognition}

2.1. How humans cognize

Think about how humans cognize before getting emotions from events. That is, in the reference (Yabu, 2020), we considered adding +1 and -1 each time individual judged the positive and negative sides of the event, but I consider how to make that judgment.

At this time, human beings divide the event into small parts and consider an example. Find rules from many examples and see if they apply to other examples. For convenience, collecting examples is +1 and applying the rules is -1 . As a result, cognition can be expressed by a one-dimensional random walk or Wiener process as in the reference (Yabu, 2020), and the average probability density function $p(x)$ of an individual can be expressed as a standard normal distribution as shown below. Understand. Here, $x$ means that the more positive it is, the more examples are collected and recognized, and the more negative it is, the more it is recognized by applying the rules. Event $X$ is given randomly, it is randomly applied on the $x$-axis of the number line, it makes a random walk toward 0 in the opposite direction, and when $x=0$, it is convinced and recognized.

$$
p(x)=\frac{1}{\sqrt{2 \pi}} e^{-\frac{x^{2}}{2}}
$$

Naturally, the probability density function of cognition as a group also has a standard normal distribution as follows. That is, the same event will be recognized with the same probability. 


$$
p_{\text {group }}(x)=\frac{1}{\sqrt{2 \pi}} e^{-\frac{x^{2}}{2}}
$$

2.2. 4 patterns that can be classified by cognition and emotion

I thought about cognition in a one-dimensional random walk. In a one-dimensional random walk, the probability of being in either the positive or negative region is much greater than the probability of being in both the positive and negative regions (Feller, 1968). That is, when humans cognize, it means that they are biased toward either collecting examples or applying rules. Also, in the reference (Yabu, 2020), human emotions were considered positive and negative, and were considered by a one-dimensional random walk. In other words, human beings are biased toward either positive thinking or negative thinking. Therefore, human beings are divided into four patterns based on cognition and emotion. That is, "Example-Positive (E-P)", "Example-Negative (E-N)", "Rule-Negative (R-N)", and "Rule-Positive (R-P)".

Positive people are not afraid to associate with others. Negative people think lonely. From this and the relationship with cognition, each type can be considered as follows. These four patterns are determined by basic human behavior such as cognition and emotions. In addition, it is considered that there is no mathematically stochastic bias in the four patterns.

\subsection{1. "Example-Positive (E-P)" type}

Collect examples in fellowship with others. Therefore, given a group goal, they will try to collect it as an example and try to achieve the goal. Also, when testing in a group, they want to collect better examples, so they will try to get good results in the test so that it will be a better example. On the other hand, even if they are given a morally bad goal, they will try to collect it as an example. It also attacks others who cannot achieve their goals or who interfere with their achievement.

\subsection{2. "Example-Negative (E-N)" type}

Collect examples lonely. Therefore, they try to classify physical phenomena and social phenomena in more detail and collect examples. As a result, they try to understand physical and social phenomena. On the other hand, if they try to collect examples toward their inner side, they will also try to collect examples from the negative side, so they will be easily depressed. Also, they will not be able to proceed with things because they are too particular about the details. 


\subsection{3. "Rule-Negative (R-N)" type}

Apply the rules to loneliness. they value their rules and try to apply their rules to various phenomena within themselves and to make their rules concrete (text, paintings, mathematical formulas, etc.). On the other hand, if they stick to their rules, they become stubborn and selfish, and as a result, they become aggressive and isolated to follow their rules.

\subsection{4. "Rule-Positive (R-P)" type}

In fellowship with others, try to apply your rules to others. Since they have the basic rule that we are positive, we want others to be positive and act accordingly. On the other hand, if they stick to applying their rules, they are willing to attack people outside the group (that is, bullying and moral harassment) in order to make the group you belong to positive. Even inside, they try to apply their rules to others without considering the inconvenience of others.

\subsubsection{For the group to function well}

For a group to function well, it is desirable that four types exist in the group. The R-N type sets goals based on his rules, the E-P type acts to achieve the goal, the E-N type assists the details, and the R-P type excites the group. It is believed that this causes the group to function well.

3. How to live normally without being excluded in a group

Living normally without being excluded in a group is very important for people who have difficulty predicting the emotions of others, such as autism spectrum disorders (so-called Asperger's syndrome). Think of a way to do that.

3.1. About the probability distribution function that expresses what the distribution of one's emotions looks like to a group

It is considered that the group excludes individuals when it is judged that they have a probability distribution function of emotions different from the probability density function of the group's emotions as an average. Therefore, consider the probability distribution function $F(z, t)$ that expresses what the probability distribution of individual's emotion looks to the group. Here $z$ means the value of emotion as in the reference (Yabu, 2020). Considering the same as the emotional probability density function in the reference, the following differential equation can be obtained for $F(z, t)$. 


$$
\frac{\partial F}{\partial t}=\frac{1}{2} \frac{\partial^{2} F}{\partial z^{2}}
$$

Finding the solution for $F(z, t)$ gives the following equation.

$$
F(z, t)=\frac{1}{\sqrt{2 \pi t}} \int_{-\infty}^{\infty} e^{-\frac{(z-y)^{2}}{2 t}} f(y) d y
$$

In order to obtain the average probability distribution function from this $F(z, t)$ and obtain a standard normal distribution, $f(y)$ should be given by the following function as an initial condition. However, $\delta(y)$ is a Dirac delta function.

$$
f(y)=\delta(y)
$$

That is, equation (4) becomes the following equation.

$$
F(z, t)=\frac{1}{\sqrt{2 \pi t}} e^{-\frac{z^{2}}{2 t}}
$$

Therefore, when the average probability distribution function is obtained using equation (6), the standard normal distribution is obtained from the reference as follows.

$$
F_{\text {mean }}(z)=\frac{1}{\sqrt{2 \pi}} e^{-\frac{z^{2}}{2}}
$$

Therefore, the $F_{\text {mean }}(z)$ observed by the group is equal to the standard normal distribution, which is the probability density function of the group's emotions. It is believed that this will prevent individuals from being excluded from the group.

\subsection{How to avoid being excluded from the group}

It is important to satisfy equation ( 5 ) in order not to be excluded from the group. That is, when an event is given by a group or the like, it has a value only at $y=0$, so it is important to always be emotionless as an initial condition. After that, individual can act according to the probability density function of individual's emotions. This is because equations ( 6) and ( 7) are functions determined by the group and have nothing to do with the probability density function of individual's emotions.

Therefore, it is important to always have an expressionless or emotionless expression with the corner of the mouth raised, and to react to it without breaking it even when an event is given. Even if you are happy, you will not react very happily, and even if you are sad, you will not react very sadly. It is important to always start reacting in neutral. If there is a request or phenomenon that you cannot keep your neutrality, you can refuse or state that you do not like it with the same expression.

Also, the probability density function of the emotions of others is not needed to prevent them from being excluded from the group. This means that you don't have to consider the feelings of others. Moreover, you do not have to consider how you should react to 
others. In the reference (Yabu, 2020), I wrote that once an event and emotion are decided, a reaction occurs like a dictionary. In other words, there is no need to take any measures against this dictionary. It may or may not be changed depending on the other party and the situation. By changing the dictionary depending on the person and the situation, I think that individual consciousness will be created.

The above method was also pointed out in cognitive-behavioral therapy (Beck, 2020), and the same results as the method derived from many cases were obtained from the mathematical approach.

4. How to overcome the difficulty of living

Some people find it difficult to live because they are negative or do not feel well about their emotions. Think mathematically about how to overcome it.

\subsection{Causes of difficulty in living}

Think about the cause of difficulty in living. As in the reference (Yabu, 2020), the probability density function of an individual's emotions has a standard normal distribution. This is the same for everyone. The problem is that the dictionary that determines the reaction to events and emotions does not work well, and the individual cannot feel one's true feelings. Therefore, it is sufficient to know that the distribution of one's emotions is a standard normal distribution.

4.2. About the probability distribution function that expresses what your emotional distribution looks like

The probability distribution function, which expresses how one's emotional distribution looks to the surroundings and events from oneself, is defined as $F(z, t)$ as in Section 3.1. At this time, equation ( 3) holds in the same way as in Section 3.1. Therefore, from $F(z, t)$, in order for the mean probability distribution function to have a standard normal distribution, $f(y)$ should be given by equation ( 5) as an initial condition.

\subsection{How to get rid of the difficulty of living}

In order to feel that the distribution of one's emotions is a standard normal distribution, since it has a value only at $y=0$ for the surroundings and events from oneself, it is important to be calm and emotionless as an initial condition. After that, you can act according to the probability density function of your emotions.

Even when you are worried or suffering, try to be calm and emotionless when you 
notice. Individual look at oneself who is suffering and suffering without thinking calmly and emotionally. After that, individual can follow the probability density function of one's emotions. By doing this, individual can feel that one's emotional distribution is a standard normal distribution.

The above method was also pointed out in cognitive-behavioral therapy (Beck, 2020), and the same results as the method derived from many cases were obtained from the mathematical approach.

\section{Conclusion}

For the psychological aspect of human beings, I applied a mathematical approach to another psychological phenomenon. First, I examined cognition before humans felt emotions, considered cognition using a one-dimensional random walk or Wiener process, and obtained a probability distribution function for cognition. As a result, it was shown that human beings can be classified into 4 types because the probability of being in one of the two types is very high for each of cognition and emotion. I also mathematically determined the characteristics of each and the conditions under which the group functions well. Next, I showed how to behave in order to live normally in a group. In order not to be excluded from the group, it is important to judge that they have the same emotional probability distribution from the group. Therefore, I considered a probability distribution function that expresses what the distribution of individual's emotions looks like to the group, found the differential equation that the probability distribution function satisfies, and found the solution. As a result, we were able to find the initial conditions that would result in a standard normal distribution, which is a probability distribution function of group emotions. As a result, we showed how the probability distribution function that looks like a group becomes the same standard normal distribution as the group. Using this result, we showed how to live normally without being excluded in the population. The method shown was also pointed out in cognitive-behavioral therapy, and the same results as those obtained from many cases were obtained. In addition, even if individual has difficulty living, individual can think mathematically in the same way as how to prevent one from being excluded from the group, and showed how individual behaves toward oneself. The method shown was also pointed out in cognitive-behavioral therapy, and the same results as those obtained from many cases were obtained.

\section{References}

Andrianov, A., Ziabari, S. S., \& Gerritsen, C. (2021). A brain-inspired cognitive support 
model for stress reduction based on an adaptive network model. Cognitive Systems Research Vol.65.

Barrya, L. C., Coman, E., Wakefield, D., Trestman, R. L., Conwell, Y., \& Steffens, D. C. (2020). Functional disability, depression, and suicidal ideation in older prisoners. Journal of Affective Disorders, Volume 266, 1 April.

Beck, S. J. (2020). Cognitive Behavior Therapy, Third Edition: Basics and Beyond. The Guilford Press.

Busemeyer, J. R., \& Diederich, A. (2002). Survey of decision field theory. Mathematical Social Sciences.

Busemeyer, J. R., \& Townsend, J. T. (1993). Decision Field Theory: A dynamic cognition approach to decision making. Psychological Review.

Feller, W. (1968). An Introduction to Probability Theory and Its Applications, Vol. I. New York-London-Sydney, John Wiley and Sons, Inc.,.

Gawad, N., Wood, T. J., Cowley, L., \& Raiche, I. (2021). How do cognitive processes influence script concordance test responses? medical education Vol. 55 Isssue 3.

Gong, B., \& Wu, C. (2021). The mediating and moderating effects of depression on the relationship between cognitive function and difficulty in activities of daily living among postmenopausal women. Menopause, Volume 28, Issue 6.

Herman, K. C., Reinke, W. M., Dong, N., \& Bradshaw, C. P. (2020). Can effective classroom behavior management increase student achievement in middle school? Findings from a group randomized trial. Journal of Educational Psychology. Advance online publication.

Kalwani, S., \& Mahesh, J. (2020). Trends in Organizational Behavior: A Systematic Review and Research Directions. Journal of Business and Management, Vol.26, No.1.

Machaena, Z., Martinb, L., \& Rosalesa, J.-H. (2021). Bio-inspired cognitive model of motor learning by imitation. Cognitive Systems Research Vol.66.

Makransky, G., \& Petersen, G. B. (2021). The Cognitive Affective Model of Immersive Learning (CAMIL): a Theoretical Research-Based Model of Learning in Immersive Virtual Reality. Educational Psychology Review. doi:https://doi.org/10.1007/s10648-020-09586-2

McLachlan, K., Flannigan, K., Temple, V., Unsworth, K., \& Cook, J. L. (2020). Difficulties in Daily Living Experienced by Adolescents, Transition-Aged Youth, and Adults With Fetal Alcohol Spectrum Disorder. ALCOHOLISM, Volume 44, Issue 8.

Misch, A., \& Dunham, Y. (2021). (Peer) Group influence on children's prosocial and 
antisocial behavior. Journal of Experimental Child Psychology, Vol. 201, 104994. doi:https://doi.org/10.1016/j.jecp.2020.104994

Monge, C. K., \& O'Brien, T. C. (2021). Effects of individual toxic behavior on team performance in League of Legends. Media Psychology. doi:https://doi.org/10.1080/15213269.2020.1868322

van der Cruijsen, C., \& Knoben , J. (2021). Ctrl+C Ctrl+Pay: Do People Mirror Electronic Payment Behavior of their Peers? Journal of Financial Services Research, Vol.59. doi:https://doi.org/10.1007/s10693-020-00345-6

Wollschlaeger, L. M., \& Diederich, A. (2020). Similarity, Attraction, and Compromise Effects: Original Findings, Recent Empirical Observations, and Computational Cognitive Process Models. The American Journal of Psychology Vol.133, No.1.

Xiaopeng, W., Rongxiu, W., Hua-Hua, C., Qiping, K., \& Yi, Z. (2020). International Comparative Study on PISA Mathematics Achievement Test Based on Cognitive Diagnostic Models. Frontier in Psychology 11:2230. doi:https://doi.org/10.3389/fpsyg.2020.02230

Yabu, T. (2020). Mathematical approach to the psychological aspects of human. https://psyarxiv.com/u3zeh/. doi:https://doi.org/10.31234/osf.io/u3zeh

Yunfei, Z., \& Smidts, C. (2021). CMS-BN: A cognitive modeling and simulation environment for human performance assessment, part 1 - methodology. Reliability Engineering \& System Safety Vol.213, 107776. 